\title{
O Estado de Saúde Individual e as Diferenças Salariais no Brasil
}

\author{
Letícia Xander Russo ${ }^{1}$ \\ Joilson Dias ${ }^{2}$
}

\begin{abstract}
Resumo: O artigo investiga as diferenças salariais segundo o estado de saúde individual no Brasil. Para identificar os fatores que mais contribuem para o diferencial salarial o método empregado consiste na decomposição de Blinder-Oaxaca, considerando o procedimento de Heckman. A condição de saúde é mensurada pela autoavaliação dos indivíduos e pela prevalência de doenças crônicas. Os resultados apontam que indivíduos saudáveis são mais bem remunerados, sendo os salários dos indivíduos que não reportaram uma boa saúde em torno de $34 \%$ a menos em relação aos que reportaram uma boa saúde, e para indivíduos que indicaram prevalência de doenças crônicas de $38 \%$ a menos em relação a aqueles que não indicaram. A escolaridade destaca-se dentre as características ao explicar parte dessa diferença salarial, contribuindo principalmente para o diferencial do grupo que autoavaliou seu estado de saúde. Contudo, fatores não explicados, que inclui a discriminação, são os que mais contribuem para a diferença de salários.
\end{abstract}

Palavras-chave: Diferenciais Salariais; Estado de Saúde; Discriminação.

1 Doutoranda em economia na Universidade Estadual de Maringá (UEM). Áreas de interesse incluem economia da saúde, capital humano e microeconometria.

2 PhD pela Universidade da Carolina do Sul. Atualmente é professor titular do departamento de economia na Univerisdade Estadual de Maringá. 


\title{
Health Status and Wage Differentials in
} Brazil

\begin{abstract}
The paper investigates wage differentials according to individual health status. In order to identify the factors that most contribute to the wage differentials, the method employed is the Blinder-Oaxaca decomposition using the Heckman correction for sample selection. The health status is measured by self-assessment of individuals and the prevalence of chronic diseases. The results show that healthy individuals have higher wages. The wages of individuals who did not report good health are around 34\% lower than those who reported good health, and for individuals who indicated prevalence of chronic are around 38\% lower compared to those who did not indicate. The educational status stands out among the characteristics explaining most of this wage differential between healthy and unhealthy, contributing mainly to the difference in salaries of the group self-reported health. However, most of the wage gap is due to unexplained factors, including discrimination.
\end{abstract}

Key-words: Wage Differentials; Health Status; Discrimination.

JEL classification: J31, J71, I10

\section{Introdução}

Nas últimas décadas o interesse em investigar a relação entre o estado de saúde com variáveis socioeconômicas se intensificou. Dentre as variáveis amplamente associadas à condição de saúde pela literatura inclui-se o rendimento. A concepção de que saúde tem impacto nos rendimentos está atrelada ao conceito de que a saúde é parte integrante do capital humano, a qual remonta ao fim da década de 50 e início da década de 60 (Mincer, 1958; Schultz, 1961; Becker, 1962).

Nesse âmbito, uma ampla literatura têm analisado quais canais contribuem para explicar a perda de rendimento de indivíduos que apresentam problemas de saúde. Bloom e Canning (2000) destacam a positiva correlação entre saúde e renda per capita, em que níveis mais elevados de escolaridade e investimento em capital físico são mecanismos que devem ser determinantes no rendimento daqueles que apresentam bom estado de saúde. Pelkowski e Berger (2004) e 
Alves e Andrade (2003) pontuam os efeitos da saúde no mercado de trabalho, os autores encontram que um pior estado de saúde implica em redução das horas trabalhadas e da probabilidade de participação na força de trabalho. Aliado a esses, a discriminação no mercado de trabalho também se destaca como um elemento relevante nas questões que norteiam o diferencial salarial. Gannon e Munley (2009) ressaltam que a discriminação de deficientes no mercado de trabalho pode ser resultante do preconceito ou de uma percepção errônea sobre a produtividade dos trabalhadores.

No Brasil, frente a marcante característica de desigualdade salarial, a estrutura do salário é comumente analisada nos mais distintos âmbitos. Em específico para a saúde, sua relação com o rendimento tem sido explorada, contudo, não da forma aqui retratada, que consiste em decompor a diferença dos salários. Decompor o diferencial dos salários permite explorar a contribuição individual de cada variável, bem como avaliar se o diferencial advém das características dos indivíduos, como escolaridade e sexo, ou se é proveniente de fatores não explicados pelas características. Fatores não explicados abrangem a discriminação no mercado de trabalho.

Nesse sentido, o presente artigo tem como objetivo analisar o diferencial salarial segundo o estado de saúde individual procurando estabelecer quais fatores que determinam tal diferença, bem como se o diferencial advém das características dos indivíduos ou é proveniente de fatores não explicados pelas características. Para tanto, se utiliza duas variáveis para mensurar a saúde: a primeira é a qualidade da saúde autorreferida pelos indivíduos e, a segunda, a presença de doenças crônicas diagnosticada.

O artigo encontra-se estruturado em mais quatro seções além desta introdução. A primeira seção apresenta uma breve revisão de estudos que abordam a relação entre saúde e rendimento. A segunda seção é referente ao método adotado e a descrição dos dados utilizados da Pesquisa Nacional de Amostra por Domicílios - PNAD 2003 e 2008. A terceira seção se destina aos resultados. Por fim, a quarta seção é composta das considerações finais.

\section{A Relação entre Saúde e Rendimento}

A literatura destaca quatro principais canais pelos quais a saúde deve ser determinante no rendimento: redução da produtividade, diminuição das horas disponíveis ao trabalho, redução do período que permanece disposto a ofertar força de trabalho e menor investimento em educação. 
Primeiro, indivíduos saudáveis são mais propensos a desenvolverem por completo suas capacidades e habilidades, ou seja, são mais aptos a possuírem uma maior força física e mental. É nesse sentido que um maior estoque de saúde deve intervir na produtividade individual. Uma expressiva literatura investiga a relevância da saúde na determinação dos ganhos individuais partindo de funções de salário/hora atrelada aos ganhos de produtividade (Gambin, 2005; Contoyannis; Rice, 2001; Alves; Andrade, 2003). Em tese, os estudos constatam a perda de rendimento de pessoas com pior condição de saúde.

Segundo, indivíduos com problemas de saúde apresentam redução nas horas de trabalho. Andrén e Palmer (2001) analisam se o histórico de doenças afeta os rendimentos anuais e o salário/hora. Os autores partem da hipótese de que se problemas de saúde interferem apenas na capacidade de trabalho, ou seja, não reduzem a produtividade, ocorreria somente restrição das horas trabalhadas. Contudo, os resultados evidenciam que ambas as variáveis são reduzidas.

Em terceiro, problemas de saúde estão também associados com limitação do período disponível despendido ao mercado de trabalho, ocasionando restrição na capacidade de ganhos (Smith, 1999). O estado de saúde individual se torna primordial na definição do tempo disponível do indivíduo, dado que pessoas saudáveis não precisam perder dias de trabalho devido a doenças e são capazes de trabalhar durante mais horas na semana e mais semanas ao longo do ano (Grossman, 1972). Tal resultado é confirmado empiricamente (Alves; Andrade, 2003; Pelkowski; Beyer, 2004).

Por último, a condição de saúde deve intervir no nível educacional. Escolaridade e saúde são componentes cruciais na teoria do capital humano, o que suscita intenso interesse em compreender como se estabelece a interação entre ambas variáveis. Fuchs (1982) denota que a educação tem se mostrado a variável mais correlacionada com a saúde, relação evidenciada, por exemplo, em Grossman (2000, 2006). Pessoas saudáveis apresentam maior frequência escolar, melhor desenvolvimento cognitivo e são produtores mais eficientes de adição de estoque de conhecimento (Shakotko; Edwards; Grossman, 1980; Grossman, 2004). A relação entre saúde e escolaridade é apontada em distintos âmbitos: abordando a conexão do peso da criança no nascimento com resultados durante a vida adulta para gêmeos (Black; Devereux; Salvanes, 2007; Oreopoulos; Stabile; Walld; Roos, 2008), choques durante o período gestacional (Almond, 2006; Almond; Edlund; Palme, 2009) transtorno de conduta na infância (Vujic et al., 2008) e nutrição durante a primeira infância (Maluccio et al., 2009).

Outra ênfase é dada pela teoria ao atribuir perda de rendimento devido a problemas de saúde, a qual se refere à discriminação no mercado de trabalho. Tal concepção é ligada a percepção da produtividade individual. Isto é, devido à condição de saúde do indivíduo, bem como deficiências apresentadas, supõe-se que sejam menos produtivos e, portanto, são mais mal 
remunerados (Contoyannis; Rice, 2001; Currie; Madrian, 1999). Baldwin e Johnson (2000) analisam as diferenças salariais de pessoas com deficiência. Os autores procuram estabelecer se a perda de rendimento dos deficientes é atribuída aos limites da condição de saúde ou se advém de discriminação entre os trabalhadores. Como resultado, encontram que as limitações físicas explicam parte do diferencial salarial entre indivíduos com e sem deficiências, contudo, ao controlar pelas diferenças das limitações físicas o diferencial de rendimento não é eliminado, ou seja, pessoas com deficiências estão sujeitas a discriminação salarial. Demais estudos constatam a perda salarial de pessoas com deficiências, atestando mediante a decomposição dos salários a forte influência da discriminação no mercado de trabalho (Hosseinpoor et al., 2012). Jones, Latreille e Sloane (2003) encontram que tal diferencial é superior para mulheres do que para homens. Jones, Latreille e Sloane (2006) destacam que a baixa participação de deficientes no mercado de trabalho é decorrente também de menor qualificação.

Esses são os principais canais destacados pela literatura ao relacionar saúde e rendimento. Contudo, a decomposição salarial aqui empregada não se limita a perda de renda devido à redução das horas disponíveis ao trabalho, uma vez que investiga os principais fatores que contribuem para explicar o diferencial do salário/hora trabalhado entre os grupos. O método é descrito a seguir.

\section{Método e Descrição dos Dados}

\subsection{A decomposição dos salários}

A decomposição de Blinder-Oaxaca (Blinder, 1973; Oaxaca, 1973) tem sido frequentemente utilizada na literatura para investigar o diferencial de salários no mercado de trabalho entre grupos, como por sexo e cor. A decomposição de Blinder-Oaxaca divide o diferencial salarial entre dois grupos em três partes. A primeira é a parte explicada pelas características dos indivíduos, como escolaridade. A segunda corresponde à parte não explicada, a qual tem sido atribuída como discriminação. Uma terceira parte pode ser incluída, a interação, a qual se refere à interação de ambas as partes.

Embora grande parcela das aplicações da decomposição se destine a discriminação no mercado de trabalho, o método também é aplicado em demais estudos com a finalidade de avaliar a relevância da parte explicada, como em 
O’Donnell et al. (2008). O autor investiga desigualdades de saúde entre os grupos pobres e não pobres, sendo possível analisar se diferenças na saúde são atribuídas ao diferencial na educação, renda, acesso e qualidade da saúde.

Para estimativa do diferencial salarial segundo o estado de saúde individual, a equação estimada é uma equação minceriana (MINCER, 1974) acrescentada de demais variáveis explicativas e considerando o método de Heckman (1979), conforme equação (1),

$$
\ln \mathrm{w}_{\mathrm{j}}=\mathrm{X}_{\mathrm{j}}^{\prime} \beta_{\mathrm{j}}+\varepsilon_{\mathrm{j}}
$$

em que $w$ representa a renda divida pelas horas de trabalho, $\mathrm{X}$ o vetor de variáveis explicativas e a constante, $\beta$ é a inclinação dos parâmetros e o intercepto e $\varepsilon$ é o termo aleatório. $j=A, B$ representa o salários dos trabalhadores dos grupos $\mathrm{A} \mathrm{e} \mathrm{B}$, nesse caso, os grupos correspondem aos indivíduo que referiram ou não uma boa saúde, e aos indivíduos com prevalência de doenças crônicas e sem prevalência de doenças crônicas.

Conforme Jann (2008), a diferença no logaritmo do salário, é a diferença do valor esperado do logaritmo do salário entre o grupos $\mathrm{A}, E\left(\ln w_{A}\right)$, e o grupo B, $E\left(\ln w_{B}\right)$.

$$
E\left(\ln w_{A}\right)-E\left(\ln w_{B}\right)=E\left(X_{A}\right)^{\prime} \beta_{A}-E\left(X_{B}\right)^{\prime} \beta_{B}
$$

Sendo que, por suposição, $E\left(\beta_{j}\right)=\beta_{j}$ e $E\left(\varepsilon_{\mathrm{j}}\right)=0$, temos,

$$
E\left(\ln w_{j}\right)=E\left(\mathrm{X}_{j}^{\prime} \beta_{j}+\quad\right)=E\left(\mathrm{X}_{j}^{\prime} \beta_{j}\right)+E\left(\varepsilon_{\mathrm{j}}\right)=E\left(\mathrm{X}_{j}\right)^{\prime} \beta_{j}
$$

Assim, para obter o diferencial total entre os grupos dividido em três partes (three-fold decomposition), é necessário realizar algumas manipulações algébricas. Na equação (1) para o grupo $A$, considere $\beta_{A}=\beta_{B}+\left(\beta_{A}-\beta_{B}\right)$ e $X_{A}=$ $X_{B}+\left(X_{A}-X_{B}\right)$. Substituindo essas expressões em (2) e rearranjando,

$$
\begin{gathered}
E\left(\ln w_{A}\right)-E\left(\ln w_{B}\right)=E\left(X_{B}\right)^{\prime} \beta_{B}+E\left(X_{B}\right)^{\prime}\left(\beta_{A}-\beta_{B}\right)+\left[E\left(X_{A}\right)-\right. \\
\left.E\left(X_{B}\right)\right]^{\prime} \beta_{B}+\left[E\left(X_{A}\right)-E\left(X_{B}\right)\right]^{\prime}\left(\beta_{A}-\beta_{B}\right)-E\left(X_{B}\right)^{\prime} \beta_{B}
\end{gathered}
$$




$$
\begin{gathered}
E\left(\ln w_{A}\right)-E\left(\ln w_{B}\right)= \\
\underbrace{\mathrm{E}\left(\mathrm{X}_{\mathrm{B}}\right)^{\prime}\left(\beta_{\mathrm{A}}-\beta_{\mathrm{B}}\right)}_{\mathrm{C}}+\underbrace{\left[\mathrm{E}\left(\mathrm{X}_{\mathrm{A}}\right)-\mathrm{E}\left(\mathrm{X}_{\mathrm{B}}\right)\right]^{\prime} \beta_{\mathrm{B}}}_{\mathrm{E}}+\underbrace{\left[\mathrm{E}\left(\mathrm{X}_{\mathrm{A}}\right)-\mathrm{E}\left(\mathrm{X}_{\mathrm{B}}\right)\right]^{\prime}\left(\beta_{\mathrm{A}}-\beta_{\mathrm{B}}\right)}_{\mathrm{I}}
\end{gathered}
$$

A primeira parte representa o "coefficients" (C), ou seja, a parte que é devido a diferenças nos coeficientes, inclusive no intercepto. Essa parte representa a valorização diferente de um mesmo atributo e, portanto, é denominada na literatura como o termo de discriminação.

A segunda parte, "endowments effects" (E), representa a parte da diferença na remuneração devido às características de cada grupo. Assim, o indivíduo é mais bem remunerado conforme suas características produtivas, como escolaridade e experiência. Esse termo é considerado como a parte explicada.

A terceira parte, "interation" (I) corresponde a um termo de interação devido a diferenças de características (E) e de coeficiente (C) serem encontradas simultaneamente em ambos os grupos.

\subsection{Variáveis Selecionadas}

A variável dependente utilizada na regressão é o logaritmo do salário/hora trabalhada. As variáveis explicativas são: escolaridade, escolaridade ${ }^{2}$, escolaridade $^{3}$, experiência e experiência ${ }^{2}$. No tocante as variáveis escolaridade e experiência é necessário esclarecer dois pontos referentes a cada.

Escolaridade: i) A escolaridade é considerada em anos de estudos, sendo de o a 15 anos, onde 15 refere-se a 15 ou mais anos de estudo. ii) A inclusão do termo da escolaridade ao quadrado e ao cubo. Em geral, a literatura considera a linearidade na taxa de retorno da educação, contudo, estudos adicionais apontam para a não linearidade dos retornos. Isto é, enquanto modelos lineares assumem que os retornos da educação são idênticos para os anos de escolaridade, os modelos não lineares permitem a existência de retornos crescentes ou decrescentes (Psacharopoulos, 1985; Heckman; Lochner; Todd, 2008). Para o Brasil, Dias et. al (2013) encontram retornos crescentes a partir de 4,7 anos de escolaridade.

Experiência: i) A experiência é calculada pela idade do indivíduo menos a idade em que começou a trabalhar. ii) A variável experiência também é incluída na forma quadrática. O objetivo é captar o comportamento decrescente das 
taxas de retorno da experiência, isto é, para níveis superiores de experiência adquirida no mercado de trabalho, a sua contribuição para o rendimento cresce, mas a taxas decrescentes (Mincer, 1974).

As demais variáveis explicativas são binárias: carta assume 1 para trabalhadores que possuem carteira assinada e o, caso contrário; mulher assume 1 para mulheres e o, caso contrário; casado assume 1 para indivíduos casados e o, caso contrário; d2008 assume 1 para o ano de 2008 e o, caso contrário; saúde assume 1 para indivíduos que avaliaram seu estado de saúde muito bom e bom, e o para indivíduos que avaliarem seu estado de saúde regular, ruim e mito ruim; doenças crônicas que assume 1 caso o indivíduo reportou 2 ou mais doenças crônicas e o, caso contrário; informante assume 1 quando o próprio indivíduo autoavaliou seu estado de saúde e/ou reportou doença(s) crônica(s) e o, caso contrário; cor quatro variáveis binárias para distinguir entre branca (base), preta, amarela e parda; região cinco variáveis binárias para distinguir as regiões Nordeste (base), Norte, Centro-Oeste, Sudeste e Sul.

Para se evitar um possível viés de seleção, no qual o indivíduo sem remuneração não está inserido no mercado de trabalho por opção própria, dado que seu salário de reserva é superior ao salário de mercado, se deve corrigir o viés com o procedimento de Heckman (1979). Assim, é considerada a equação de seleção (select) em que a variável dependente é indivíduo trabalha e as variáveis explicativas são escolaridade, casado, número de membros da família, mulher com filho menor de 14 anos de idade e indivíduo está buscando emprego.

\subsection{Descrição dos Dados}

Os dados utilizados são da PNAD - Pesquisa Nacional por Amostra de Domicílios - para o ano de 2003 e 2008, os quais apresentam suplemento especial sobre saúde.

Para mensurar o estado de saúde do indivíduo se adotou dois critérios de acordo com as questões disponíveis da PNAD 2003 e 2008. O primeiro critério refere-se à autoavaliação do indivíduo do seu estado de saúde, no qual o indivíduo classifica sua saúde como muito boa, boa, regular, ruim e muito ruim.

O segundo critério adotado foi a prevalência de doença crônica, na qual o indivíduo responde no questionário da PNAD se tem ou não problemas de saúde de natureza crônica, sendo considerado no presente trabalho 12 doenças crônicas abordadas pela PNAD, a saber: coluna, reumatismo, câncer, diabetes, bronquite, hipertensão, coração, renal, depressão, tuberculose, cirrose e tendinite. Criou-se uma variável que identifica indivíduos com prevalência 
de duas ou mais doenças crônicas (doenças crônicas).

Dessa forma, como encontrado na literatura empírica, adotamos dois indicadores do estado de saúde dos indivíduos: o subjetivo, que consiste na autoavaliação do seu estado de saúde e; indicadores do estado de saúde clínico individual, que consiste na presença de doença(s) crônica(s).

Para o presente trabalho foram considerados indivíduos entre 20 e 64 anos que apresentem um salário/hora trabalhada de até $\mathrm{R} \$ 600,00$. O filtro do rendimento objetiva excluir da amostra indivíduos com salário extremamente alto, que poderiam enviesar os resultados. O Distrito Federal, por ter sua atividade econômica concentrada no setor público, foi excluído da amostra. Nas regressões foram considerados também os pesos de amostragem e estratificação, devido ao plano de amostra complexa da PNAD.

A amostra é composta por 49.06\% de homens e 50.94\% de mulheres; $51.85 \%$ brancos, 40.44\% pardos, 7.15\% pretos e 0.56\% amarelos. Quanto à região, 6.55\% dos indivíduos são da região do Norte, $25.73 \%$ do Nordeste, $6.15 \%$ do Centro-Oeste, $46.21 \%$ do Sudeste e $15.36 \%$ do Sul. Dos indivíduos da amostra, $33.68 \%$ possuem carteira assinada.

Da população, 25\% não referiu seu estado de saúde como bom; 29\% declarou sofrer de algum problema crônico de saúde, enquanto 15\% declarou sofrer de pelo menos dois dos problemas crônicos considerados. A maior incidência de doença crônica ocorre com doenças de coluna, seguida de hipertensão, reumatismo e depressão.

\section{Resultados e Discussão}

No tocante a saúde e o grau educacional, a relação entre ambas variáveis é bem estabelecida pela literatura. A associação é notória tanto para indivíduos segundo a saúde autorreferida como pelo diagnóstico de doenças crônicas, como exposto no gráfico 1. 
GRÁFICO 1: PROPORÇÃO DE INDIVÍDUOS POR ANO DE ESCOLARIDADE SEGUNDO O ESTADO DE SAÚDE

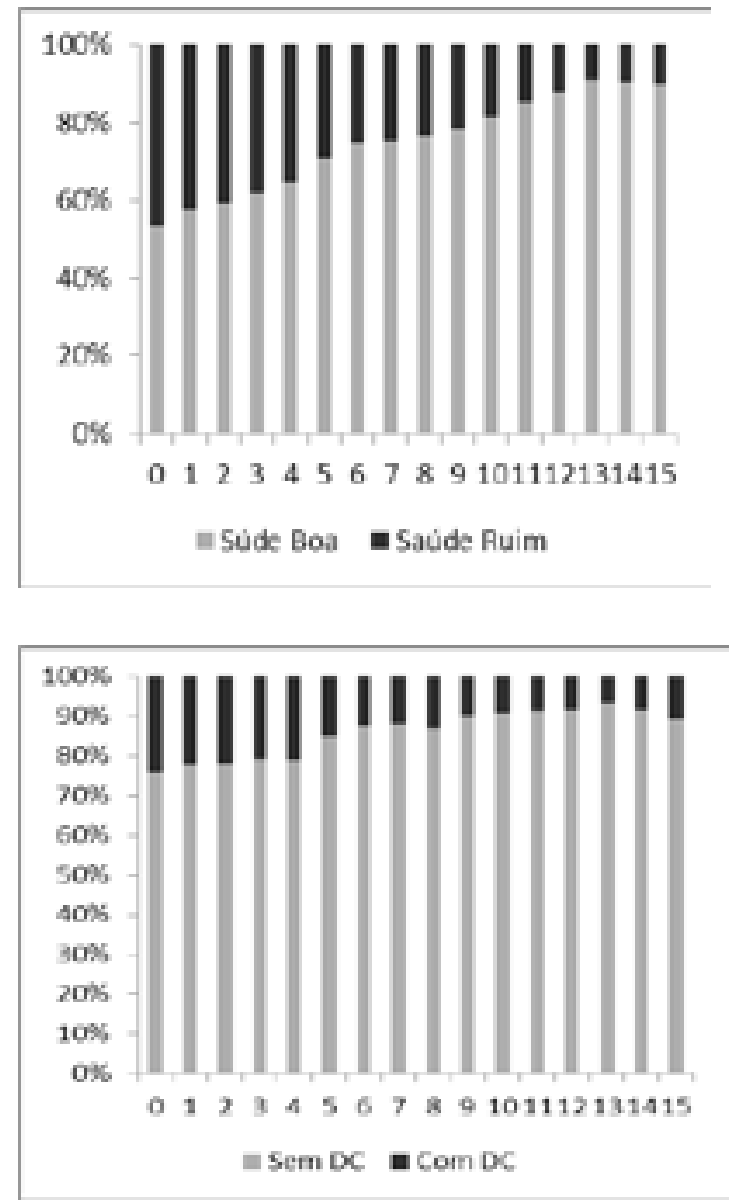

Fonte: PNAD - 2003 e 2008. Elaboração dos autores.

Dos indivíduos sem escolaridade 46\% não referiram uma boa saúde, enquanto dos indivíduos com 15 anos ou mais de escolaridade essa quantidade cai para 10\%. Para a proporção de indivíduos com e sem problemas crônicos se verifica que há maior concentração de indivíduos com prevalência de doenças crônicas para até 4 anos de escolaridade, sendo que em média $22 \%$ dos indivíduos das séries iniciais (o-4) referiram problemas crônicos de saúde.

Nesse aspecto, devido à relação entre as referidas variáveis, o nível educacional se torna extremamente relevante para a análise do diferencial de renda, uma vez que a escolaridade apresenta forte influencia nos rendimentos. Ademais, demais fatores também devem atuar como determinantes no diferencial 
salarial.

Isto posto, os resultados do método empregado estão expostos a seguir segundo o critério para mensurar a condição de saúde individual, inicialmente para saúde autorreferida e em seguida para doenças crônicas diagnosticadas.

\subsection{Saúde Autorreferida}

Com base na decomposição de Blinder-Oaxaca, os resultados da tabela 1 reportam as diferenças salariais em três partes. A média do logaritmo do salário/hora é de 0.996 para indivíduos que não referiram uma boa saúde e de 1.502 para indivíduos que referiram boa saúde, apresentando um diferencial de salário de 0.5064 entre os grupos.

TABELA 1: DECOMPOSIÇÃO DE BLINDER-OAXACA - SAÚDE AUTORREFERIDA

\begin{tabular}{lcc}
\hline Saúde Ruim & $\begin{array}{c}0.996^{* * *} \\
(0.0241)\end{array}$ \\
Saúde Boa & $1.502^{* * *}$ & \\
& $(0.00677)$ & \\
Diferença & $-0.5064^{* * *}$ & $100 \%$ \\
& $(0.0242)$ & \\
Características & $-0.236^{* * *}$ & \\
& $(0.00525)$ & $47 \%$ \\
Coeficientes & $-0.255^{* * *}$ & \\
& $(0.0213)$ & $50 \%$ \\
Interação & $-0.0153^{* * *}$ & $3 \%$ \\
Observações & $(0.0046)$ & \\
\hline
\end{tabular}

Fonte: PNAD - 2003 e 2008. Elaboração dos autores. Erro padrão em parênteses ${ }^{* * *} \mathrm{p}<0.01,{ }^{* *} \mathrm{p}<0.05$, ${ }^{*} \mathrm{p}<0.1$

A parte devida às características corresponde a $47 \%$ da diferença salarial encontrada entre os grupos, a parte devida aos coeficientes corresponde a 50\% e a parte devido à interação a 3\%. Dessa forma, em torno da metade do diferencial salarial entre os indivíduos que referiram ou não boa saúde resultam de fatores explicados como escolaridade, experiência e gênero, enquanto a outra metade do diferencial de salários advém de fatores não explicados por características.

O efeito característica reflete o aumento médio no logaritmo do salário/hora 
dos indivíduos saudáveis se estes tivessem as mesmas características dos indivíduos não saudáveis. Nesse caso, o sinal negativo indica que haveria perda do salário por parte dos trabalhadores que referiram boa saúde caso tivessem as mesmas características dos trabalhadores que não referiram boa saúde.

No entanto, $50 \%$ da diferença salarial entre os dois grupos permanece não explicada pelos atributos pessoais. Sendo que, a parte não explicada pelos coeficientes denota a mudança no salário do grupo dos trabalhadores que avaliaram sua saúde como boa quando aplicado os coeficientes dos trabalhadores que avaliaram a sua saúde como ruim para as características do primeiro grupo. Ademais, o diferencial advindo dos coeficientes é descrito na literatura como proveniente da discriminação no mercado de trabalho, ou seja, ainda que com as mesmas características dos indivíduos que referiram boa saúde, aqueles que referiram saúde ruim continuam a apresentar perda salarial.

Quanto à contribuição individual das variáveis, a educação foi a que mais contribuiu para o aumento da diferença salarial entre os grupos, com $61 \%$ (Tabela 2). Contudo, observa-se que a escolaridade explicada, estatisticamente significante em nível de confiança de $95 \%$, é responsável por metade do diferencial salarial entre os grupos, ou seja, é a diferença educacional que mais contribui para a desigualdade dos salários dos trabalhadores que referiram ou não boa saúde. Os dados apontam que indivíduos que não avaliaram sua saúde como boa possuem em média 5.7 anos de escolaridade enquanto indivíduos que avaliaram sua saúde como boa apresentam em média 8.2 anos de escolaridade, portanto, sendo esta a principal variável para o diferencial de salários entre os grupos. 
RUSSO, L. X.; DIAS, J. O Estado de Saúde Individual e as Diferenças Salariais no Brasil em 2003 e 2008

TABELA 2: CONTRIBUIÇÃO DOS GRUPOS DAS VARIÁVEIS NA DECOMPOSIÇÃO DE BLINDER-OAXACA - SAÚDE

\begin{tabular}{lcccccccc}
\hline & \multicolumn{2}{c}{ Características } & \multicolumn{2}{c}{ Coeficientes } & \multicolumn{2}{c}{ Interação } & \multicolumn{2}{c}{ Total } \\
\hline Escolaridade & -0.2520 & $50 \%$ & -0.0588 & $12 \%$ & 0.0218 & $-4 \%$ & -0.3108 & $61 \%$ \\
Experiência & 0.0950 & $-19 \%$ & -0.1074 & $21 \%$ & -0.0174 & $3 \%$ & -0.0124 & $2 \%$ \\
Carta & -0.0163 & $3 \%$ & 0.0779 & $-15 \%$ & -0.0229 & $5 \%$ & 0.0616 & $-12 \%$ \\
Região & -0.0383 & $8 \%$ & 0.0011 & $0 \%$ & 0.0016 & $0 \%$ & -0.0372 & $7 \%$ \\
Mulher & -0.0101 & $2 \%$ & 0.0069 & $-1 \%$ & 0.0006 & $0 \%$ & -0.0033 & $1 \%$ \\
Casado & 0.0005 & $0 \%$ & -0.0184 & $4 \%$ & 0.0001 & $0 \%$ & -0.0179 & $4 \%$ \\
Cor & -0.0182 & $4 \%$ & 0.0072 & $-1 \%$ & 0.0019 & $0 \%$ & -0.0110 & $2 \%$ \\
d20o8 & 0.0018 & $0 \%$ & 0.0155 & $-3 \%$ & 0.0004 & $0 \%$ & 0.0173 & $-3 \%$ \\
Informante & 0.0015 & $0 \%$ & -0.0128 & $3 \%$ & -0.0015 & $0 \%$ & -0.0113 & $2 \%$ \\
Constante & & & -0.1661 & $33 \%$ & & $0 \%$ & -0.1661 & $33 \%$ \\
\hline TOTAL & -0.236 & $47 \%$ & -0.255 & $50 \%$ & -0.0153 & $3 \%$ & -0.5064 & $100 \%$ \\
\hline
\end{tabular}

Fonte: PNAD - 2003 e 2008. Elaboração dos autores.

Regiões e experiência também têm contribuído para o diferencial de salários. A experiência está associada à idade e, portanto, dado que indivíduos com pior saúde apresentam experiência média superior que o outro grupo, 29 anos e 21 anos de experiência respectivamente, se justifica o sinal negativo na parte explicada (-19\%). Considerando a parte explicada, não explicada e a interação, a variável d2008, que representa uma binária para diferenciar os anos de 2003 e 2008, indicou que não houve diferença significativa nas respostas dos indivíduos entre os períodos (-3\%). Ser casado, mulher e cor também não apresentou forte poder explicativo para o diferencial de salários entre os grupos.

A constante reflete o grupo de referência, de modo que mulheres casadas brancas sem escolaridade e experiência com carteira assinada e que residem no Nordeste seriam mais bem remuneradas caso tivessem uma boa saúde.

Sendo assim, os resultados encontrados confirmam a perda de rendimento dos trabalhadores que não referiram uma boa saúde em relação aos demais, constatando a educação média como a característica com principal contribuição para o diferencial de salários entre os grupos.

\subsection{Doenças Crônicas}

Conforme a decomposição de Blinder-Oaxaca a média do logaritmo do salário/ 
hora para indivíduos sem pelo menos dois problemas crônicos de saúde é de 1.39, enquanto de indivíduos com pelo menos dois problemas crônicos é de o.86, apresentando uma diferença salarial de 0.53 .

Da diferença de salários encontrada entre os grupos, 95\% é devido aos coeficientes e -12\% a interação (tabela 3). A parte do diferencial de salários explicada pelas características é de $17 \%$, indicando que a diferença de ganhos entre indivíduos com e sem problemas crônicos de saúde se deve principalmente a diferenças não explicadas pelas características.

\section{TABELA 3: DECOMPOSIÇÃO DE BLINDER-OAXACA - DOENÇAS CRÔNICAS}

\begin{tabular}{lll}
\hline Sem DC & $1.394^{* * *}$ & \\
& $(0.0072)$ & \\
Com DC & $0.865^{* * *}$ & \\
& $(0.0297)$ & \\
Diferença & $0.529^{* * *}$ & $100 \%$ \\
& $(0.0292)$ & \\
Características & $0.091^{* * *}$ & $17 \%$ \\
& $(0.0079)$ & \\
Coeficientes & $0.501^{* * *}$ & $95 \%$ \\
& $(0.0288)$ & \\
Interação & $-0.064^{* * *}$ & $-12 \%$ \\
& $(0.0056)$ & \\
Observações & 405,400 & \\
\hline
\end{tabular}

Fonte: PNAD - 2003 e 2008. Elaboração dos autores. Erro padrão em parênteses ${ }^{* * *} \mathrm{p}<0.01,{ }^{* *} \mathrm{p}<0.05$, ${ }^{*} \mathrm{p}<0.1$

A parte dos coeficientes tem uma elevada contribuição atribuída a diferentes critérios de remuneração adotados e que é interpretada como indicador de discriminação no mercado de trabalho. Ressalta-se ainda que a parte dos coeficientes pode refletir potencial efeito das diferenças em variáveis não observadas.

A discriminação salarial devido à condição de saúde, mensurada pelo diagnóstico de doença ou deficiência, é constatada em distintos países. Para tais trabalhadores, ainda que reportem que sua condição de saúde não produz restrição nas suas atividades diárias e, portanto, não há perda de produtividade, verifica-se que menor remuneração perdura entre eles (Gannon; Munley, 2009; JONES, 2006; Kidd; Sloane; Ferko, 2000; Bell; Heitmueller, 2009).

A despeito das diferenças nas habilidades e características produtivas obser- 
RUSSO, L. X.; DIAS, J. O Estado de Saúde Individual e as Diferenças Salariais no Brasil em 2003 e 2008

vadas dos indivíduos, estas tem menor contribuição percentual no diferencial do logaritmo do salário/hora entre pessoas com e sem problemas crônicos, do que no caso da saúde autorreferida.

Na contribuição individual das variáveis a escolaridade foi estatisticamente significante apenas na parte explicada. Embora a escolaridade ajude a explicar o diferencial salarial, sua contribuição é bem inferior do que para saúde autorreferida (tabela 4). Contudo, embora não tão expressivo, indivíduos com problemas crônicos também apresentam menor escolaridade em relação aos demais indivíduos, 6 anos e 7.8 anos de estudo respectivamente.

TABELA 4: CONTRIBUIÇÃO DOS GRUPOS DAS VARIÁVEIS NA DECOMPOSIÇÃO DE BLINDER-OAXACA - DOENÇAS CRÔNICAS

\begin{tabular}{lcccccccc}
\hline & Características & \multicolumn{2}{c}{ Coeficientes } & \multicolumn{2}{c}{ Interação } & \multicolumn{2}{c}{ Total } \\
\hline Escolaridade & 0.1340 & $25 \%$ & -0.1080 & $-20 \%$ & -0.0237 & $-4 \%$ & 0.0260 & $5 \%$ \\
Experiência & -0.0930 & $-18 \%$ & 0.1029 & $19 \%$ & -0.0225 & $-4 \%$ & 0.0099 & $2 \%$ \\
Carta & 0.0215 & $4 \%$ & -0.0319 & $-6 \%$ & -0.0079 & $-1 \%$ & -0.0104 & $-2 \%$ \\
Região & -0.0132 & $-2 \%$ & 0.0162 & $3 \%$ & -0.0009 & $0 \%$ & 0.0030 & $1 \%$ \\
Mulher & 0.0390 & $7 \%$ & 0.0160 & $3 \%$ & -0.0040 & $-1 \%$ & 0.0549 & $10 \%$ \\
Casado & 0.0026 & $0 \%$ & -0.0084 & $-2 \%$ & -0.0003 & $0 \%$ & -0.0058 & $-1 \%$ \\
Cor & -0.0024 & $0 \%$ & 0.0029 & $1 \%$ & 0.0001 & $0 \%$ & 0.0006 & $0 \%$ \\
d20o8 & 0.0015 & $0 \%$ & 0.0017 & $0 \%$ & 0.0000 & $0 \%$ & 0.0032 & $1 \%$ \\
Informante & 0.0013 & $0 \%$ & 0.0205 & $4 \%$ & -0.0045 & $-1 \%$ & 0.0218 & $4 \%$ \\
Constante & & & 0.4893 & $93 \%$ & & $0 \%$ & 0.4893 & $93 \%$ \\
\hline TOTAL & 0.0913 & $17 \%$ & 0.5012 & $95 \%$ & -0.0635 & $-12 \%$ & 0.5290 & $100 \%$ \\
\hline
\end{tabular}

Fonte: PNAD - 2003 e 2008. Elaboração dos autores.

A variável experiência é importante por estar associada à idade dos indivíduos, visto que problemas de saúde são positivamente relacionados ao avanço da idade. A experiência, estatisticamente significante em nível de confiança de 95\% e com elevada contribuição para características e coeficientes, atua na parte das características em favor do salário de indivíduos com problemas crônicos, dado que o salário é superior para os mais experientes e o sinal é negativo. Sendo que a experiência é superior para o grupo de indivíduos com doenças crônicas, em média de 32 anos para indivíduos com problemas crônicos e de 21 anos para indivíduos sem problemas crônicos de saúde.

Ser mulher tem uma influência positiva no diferencial salarial entre os grupos, enquanto ser casado e possuir carteira assinada têm reduzido às diferenças salariais. A constante apresenta uma importante contribuição para o diferencial 
entre os grupos, denotando que mulheres casadas brancas sem escolaridade e experiência com carteira assinada e que residem no Nordeste seriam mais bem remunerados se não apresentassem prevalência de doenças crônicas.

Contudo, se ressalta a importância de estudos que investiguem em separado cada uma das doenças crônicas, a fim de avaliar se há diferencial salarial entre indivíduos que indiquem ou não alguma doença específica. Uma vez que há diferença de sintomas, tratamento e intensidade entre as próprias doenças.

Em suma, os resultados apontam que, em geral, há um menor salário de indivíduos com problemas de saúde, tanto para saúde autorreferida como para prevalência de doenças crônicas, em que a educação é a principal característica para explicar esse diferencial entre indivíduos saudáveis e não saudáveis. Tal resultado é decorrente da escolaridade média dos grupos, a qual é superior entre indivíduos saudáveis, denominado como efeito de médias. Contudo, fatores não explicados ainda são responsáveis pela maior parte da diferença salarial.

Os resultados aqui auferidos são condizentes com os encontrados na literatura. Trabalhos empíricos evidenciam a importância da escolaridade no diferencial de renda, em que indivíduos mais educados apresentam retornos crescentes (Dias Et Al., 2013; Araújo; Silveira Neto, 2004). Ademais, menor salário/ hora é encontrado entre indivíduos que apresentam pior condição de saúde, resultado apontado em estudos utilizando base de dados de distintos países (Jackle, 2007; Cai, 2007; Andrén; Palmer, (2001); Kassouf, 1997), tanto ao mensurar saúde mediante a qualidade autorreferida como pelo diagnóstico de doenças crônicas (Balbinotto Neto; Godoy; Ribeiro, 2007; Silva, 2011).

\section{Considerações Finais}

No Brasil, 25\% dos indivíduos entrevistados avaliaram seu estado de saúde como regular ou ruim e $15 \%$ indicaram problemas crônicos de saúde. Isso remete a uma significativa parcela da população, que além de perda de bem-estar, são plausíveis de apresentarem pior condição econômica. Tal relação é estabelecida na medida em que se evidencia a positiva associação entre o estado de saúde individual e a renda. Não obstante, a correlação entre as mesmas é determinada por diversos canais. Nesse âmbito, buscou-se analisar o diferencial salarial segundo o estado de saúde individual enfatizando os principais fatores que contribuem para tal diferença. 
Os resultados encontrados corroboram com a literatura ao apontar que indivíduos saudáveis apresentam salário/hora superior em relação aos indivíduos com problemas de saúde. Os salários médios são em torno de $34 \%$ a menos para indivíduo que reportaram uma saúde ruim e de $38 \%$ a menos para indivíduos que indicaram problemas crônicos. Portanto, além da redução da renda mensal devido ao menor número de horas despendidas ao trabalho, é evidenciado que indivíduos não saudáveis recebem também menor remuneração por hora trabalhada.

Para indivíduos que autoavaliaram seu estado de saúde, encontrou-se que metade do diferencial salarial entre os que referiram ou não uma boa saúde deve-se a fatores explicados, estando fortemente associada ao nível de escolaridade. O nível educacional também é relevante para explicar as diferenças de salários entre indivíduos com e sem problemas crônicos, ainda que em magnitude inferior.

Contudo, fatores não explicados, dentre os quais se inclui a discriminação, contribui para a maior parte do diferencial salarial, em que se constata a desvalorização no mercado de trabalho de indivíduos com problemas de saúde. Para a saúde autorreferida a parte não explicada representa 50\% do diferencial salarial entre os grupos e 95\% para doenças crônicas.

\section{Referências}

Almond, D. (2006). "Is the 1918 Influenza pandemic over? Long-term effects of in utero influenza exposure in the post-1940 U.S. population”. Journal of Political Economy. v.114(4), pp. 672-712.

Almond, D.; Edlund, L.; Palme M. (2009). "Chernobyl's subclinical legacy: Prenatal exposure to radioactive fallout and school outcomes in Sweden”. Quartely Journal of Economics, v. 124(4), pp. 1729-1772.

Alves, L,F.; Andrade, M.V. (2003). "Impactos da Saúde nos Rendimentos Individuais no Brasil”. Economia Aplicada, v. 7, pp. 359-388.

Andrén, D; Palmer, E. (2001). “The effect of sickness on earnings". Working Paper in Economics, n.45, Department of Economics, Göteborg University.

Araújo, I.; Silveira Neto, R.M. (2004) “ “Concentração Geográfica de Capital Humano, Ganhos de Produtividade e Disparidades Regionais: evidências para o Brasil Metropolitano". Revista Econômica do Nordeste, Fortaleza, v. 35(3).

Balbinotto Neto, G.; Godoy, M.R.; Ribeiro, E.P. (2007). "Earnings and Chronic Renal Disease”. Transplantation Proceedings, v. 39 (2), pp. 378-380.

Baldwin, M.; Johnson, W. (2000). "Labor Market Discrimination Against Men with Disabilities in the Year of the ADA". Southern Economic Journal, v. 66(3), pp. 548-566. 
Becker, G.S. (1962). "Investment in Human Capital: A Theoretical Analysis". The Journal of Political Economy, v.70 (5).

Bell, D.; Heitmueller, A. (2009). "The Disability Discrimination Act in the UK: Helping or hindering employment among the disabled?”. Journal of Health Economics, v. 28, pp. 465-480.

Black, S.E; Devereux, P.J.; Salvanes, K.G. (2007). "From the cradle to the labor market? The effect of birth weight on adult outcomes". Quartely Journal of Economics, v. 122(1), pp. 409-439.

Blinder, A.S. (1973). "Wage Discrimination: Reduced form and structural estimates". Journal of Human Resources, v. 8, pp. 436-455.

Bloom, D; Canning, D. (2000). "The Health and Wealth of Nations". Science, n.287.

Cai, L. (2007). "Effects of Health on Wages of Australian Men". Working Paper. Melbourne Institute of Applied Economic and Social Research - The University of Melbourne, v. 2.

Contoyannis, P.; Rice, N. (2001). “The Impact of Health on Wages: Evidence from the British Household Panel Survey". Empirical Economics, v. 26, pp. 599-622.

Currie. J; Madrian, B.C. (1999). "Health, Health insurance and the labour Market". In: Ashenfelter O, Card D, editors. Handbook of Handbook of Amsterdam: Elsevier Science B.V., pp. 3309-3415.

Dias, J.; Monteiro, W.F.M.; Dias, M.H.A.; Russo, L.X. (2013). "Função de Capital Humano dos Estados Brasileiros: retornos crescentes ou decrescentes da educação?”. Pesq. Plan. Econ, v. 43(2).

Fuchs, V.R. (1982). “Time preference and health: an exploratory study”. In: Fuchs VR, org. Economic Aspects of Health, Chicago: University of Chigago Press.

Gambin, L.M. (2005). “The Impact of Health on Wages in Europe - Does Gender Matter?" HEDG Working Paper.

Gannon, B; Munley, M. (2009). "Age and disability: Explaining the wage differential”. Social Science \& Medicine. v. 69, pp. 47-55.

Grossman, M. (1972). "On the concept of health capital and demand for health". Journal of Political Economy, Chicago. 1972; 80 (2): 235-255.

Grossman, M. (2000). “The Human Capital Model”. In: Culyer A J, Newhouse JP, org. Handbook of Health Economics, Elsevier Science, v. 1.

Grossman, M. (2004). “The demand for health, 30 years later: a very personal retrospective and prospective reflection”. Journal of Health Economics, v. 23, pp. 629-636.

Grossman, M. (2006). "Education and Nonmarket Outcomes”. In: Hanushek E, Welch F, org. Handbook of the Economics of Education, North-Holland: Amsterdam.

Heckman, J. (1979). “Sample selection bias as a specification error”. Econometrica, pp. 153-161.

Heckman, J. J.; LOCHNER, L.; TODD, P. (2008). "Earnings functions and rates of return”. Journal of Human Capital. 2008, v. 2(1), pp. 1-31.

Hosseinpoor, A.R., et al. (2012). "Social determinants of sex differences in disability 
RUSSO, L. X.; DIAS, J. O Estado de Saúde Individual e as Diferenças Salariais no Brasil em 2003 e 2008

among older adults: a multi-country decomposition analysis using the World Health Survey”. International Journal for Equity in Health, v. 11(52).

Jackle, R. (2007). "Health and Wages Panel data estimates considering selection and Endogeneity". Ifo Working Paper. Ifo Institute for Economic Research at the University of Munich, v. 43.

Jann, B. (2008). “The Blinder-Oaxaca decomposition for linear regression models". The Stata Journal, v. 8(4), pp; 453-479.

Jones, M.K. (2006). “Is there employment discrimination against the disabled?". Economics Letters, v. 92, pp. 32-37.

Jones, M.K.; Latreille, P.L.; Sloane, P.J. (2003). “Disability, Gender and the Labour Market”. Working Paper, IZA Discussion paper series 936.

Jones, M.K.; Latreille, P.L.; Sloane, P.J. (2006). “Disability, Gender and the Labour Market in Wales”. Regional Studies, v. 40 (8), pp. 823-845.

Kassouf, A.L. (1997). "Saúde e Mercado de Trabalho”. Pesq. Plan. Econ, v. 27(3), pp. 587-610.

Kidd, M.P.; Sloane, P.J.; Ferko, I. (2000). "Disability and the labour market: an analysis of Britsh males”. Journal of Health Economics, v. 19, pp. 961-981.

Maluccio, J.A.; Hoddinott, J., Behrman, J.R.; Martorell, R.; Quisumbing, A.R.; Stein, A.D. (2009). "The impact of nutrition during early childhood on education among Guatemalan adults". The Economic Journal, v. 119(537), pp. 734-763.

Mincer, J. (1958). "Investment in human capital and personal income distribution". The Journal of Political Economy, p. 281-302.

Mincer, J. (1973). Schooling, experience, and earnings. National Bureau of Economic Research, Columbia University Press, New York.

Oaxaca, R. (1973). "Male-female wage differentials in urban labor markets". International Economic Review, v. 14, pp. 693-709.

O’Donnell, O.; Doorslaer, E. Van; Wagstaff, A.; Lindelow, M. (2008). “Analyzing Health Equity Using Household Survey Data: A Guide to Techniques and Their Implementation”. WBI Learning Resources Series. The World Bank Washington, DC.

Oreopoulos, P.; Stabile, M.; Walld, R.; Roos, L. (2008). "Short, médium, and long-term consequences of poor infant health: An analysis using siblings and twin". Journal of Human Resources, v. 43(1).

Pelkowski, J. M.; Beyer, M. (2004). "The impact of health on employment, wages, and hours worked over the life cycle". The Quartely Review of Economics and Finance, v. 44, pp. 102-121.

Psacharopoulos, G. (1985). "Returns to Education: A Further International Update and Implications". Journal of Human Resources, v. 20 (4).

Schultz, T. (1961). "Investment in human capital”. The American Economic Review, pp. 1-17.

Shakotko. R. A.; Edwards, L. N.; Grossman, M. (1980). "An exploration of the dynamic relationship between health and cognitive development”. Working Paper Series, National Bureau of Economic Research - NBER, v. 454. 
Silva, F. M. (2011). Um Estudo Econômico da Tuberculose no Brasil. 2011. Dissertação. Rio Grande do Sul: Programa de Pós-Graduação em Economia do Desenvolvimento da Faculdade de Administração, Contabilidade e Economia, Pontifícia Universidade Católica do Rio Grande do Sul.

Smith, J. P. (1999). "Healthy Bodies and Thick Wallets: The Dual Relation Between Health and Economic Status". Journal of Economic Perspectives, v. 13(2), pp. 145-166.

Vujic, S., Koning, P., Webbink, D.; Martin, N. (2008). "The effect of childhood conduct disorder on human capital”. Discussion Paper 3646. CPB Netherlands Bureau for Economic Policy Analysis. 